\title{
Development of Stabilized Growth Factor- Loaded Hyaluronate- Collagen Dressing (HCD) matrix for impaired wound healing
}

Seong Mi Choi ${ }^{1,2}$, Hyun Aae Ryu ${ }^{2}$, Kyoung-Mi Lee ${ }^{2}$, Hyun Jung Kim³ ${ }^{3}$ Ik Kyu Park ${ }^{3}$, Wan Jin Cho ${ }^{3}$, Hang-Cheol Shin ${ }^{4}$, Woo Jin Choi ${ }^{2}$ and Jin Woo Lee ${ }^{1,2^{*}}$

\begin{abstract}
Background: Diabetes mellitus is a disease lack of insulin, which has severely delayed and impaired wound healing capacity. In the previous studies, various types of scaffolds and growth factors were used in impaired wound healing. However, there were several limitations to use them such as short half-life of growth factors in vivo and inadequate experimental conditions of wound-dressing material. Thus, our study aimed to determine the biocompatibility and stability of the matrix containing structurally stabilized epidermal growth factor (S-EGF) and basic fibroblast growth factor (S-bFGF).

Results and Discussion: We stabilized EGF and bFGF that are structurally more stable than existing EGF and bFGF. We developed biocompatible matrix using S-EGF, S-bFGF, and hyaluronate- collagen dressing (HCD) matrix. The developed matrix, S-EGF and S-bFGF loaded on HCD matrix, had no cytotoxicity, in vitro. Also, these matrixes had longer releasing period that result in enhancement of half-life. Finally, when these matrixes were applied on the wound of diabetic mice, there were no inflammatory responses, in vivo. Thus, our results demonstrate that these matrixes are biologically safe and biocompatible as wound-dressing material.

Conclusions: Our stabilized EGF and bFGF was more stable than existing EGF and bFGF and the HCD matrix had the capacity to efficiently deliver growth factors. Thus, the S-EGF and S-bFGF loaded on HCD matrix had improved stability. Therefore, these matrixes may be suitable for impaired wound healing, resulting in application of clinical treatment.
\end{abstract}

Keywords: Stabilized growth factor, HCD matrix, Impaired wound healing

\section{Background}

The wound healing process consists of inflammation, contraction, neoangiogenesis, extracellular matrix deposition, granulation tissue synthesis, re-epithelialization and remodeling. These stages are related in various cellular and molecular signals [1] and when these signals are impaired, the wound healing process is delayed. This phenomenon is caused by the various form of host impairment such as malnutrition, infection and diabetes [2].

\footnotetext{
*Correspondence: ljwos@yuhs.ac

'Brain Korea 21 PLUS Project for Medical Science, Yonsei University, Seoul, South Korea

2Department of Orthopaedic Surgery, Yonsei University College of Medicine, Seoul, South Korea

Full list of author information is available at the end of the article
}

Recently, several studies have reported that the growth factors, as therapeutical agents, are widely used in impaired wound healing $[3,4]$. Platelet derived growth factor (PDGF), basic fibroblast growth factor (FGF) and epidermal growth factor (EGF) have been most prevalently studied growth factors [5-7]. Indeed, these growth factors are known as stimulating cell proliferation, recruiting various cell types into injured site, and inducing synthesis of extracellular matrix during wound healing process [8-10]. Particularly, the bFGF has ability to promote the wound repair and angiogenesis [11-13] and the EGF contributes to the wound healing via stimulating proliferation and migration of keratinocytes and also facilitating dermal regeneration, in vivo [8]. 
Although these growth factors have therapeutic effects in diabetic wound healing, they have been limited to use as therapeutic agents due to the short half-life of growth factors, in vivo. Indeed, the inadequate experimental conditions that did not continuously release growth factors from scaffolds and lose their activity readily when they are loaded onto various types of scaffolds [7, 14-17]. Thus, the new approaches are required for the stable delivery of growth factors locally into the impaired wounds.

There have been variety types of scaffolds such as collagen sponge [18], photo-crosslinking chitosan hydrogel [19] and gelatin sponge [20] which were used to deliver bFGF and EGF onto the diabetic wounds. However, these scaffolds had certain unsatisfied features including rapid absorption and poor mechanical strength [21, 22]. To compensate the shortcomings of the existing wounddressing materials, the development of new wounddressing material is necessary.

The hyaluronate- collagen dressing (HCD) matrix has been already used as wound-dressing materials because of advantages such as haemostatic effect, reduction of pain, maintenance of moisturizing condition, and feasible to exchange the wound dressing material because the surface of the HCD matrix does not adhere to wound. For these reason, we considered that HCD matrix is suitable for base material. So, we have assessed the biocompatibility and stability of modified growth factor-loaded HCD matrix, in vitro. Furthermore, we investigated in vivo stability of modified growth factors-loaded HCD matrix, via application on the diabetic mice.

\section{Methods}

\section{Materials}

Hyaluronic acid (Shisheido, Shizuoka, Japan), Collagen (Koken, Tokyo, Japan) and Pluronic F68 (Daebong LS, Incheon, Korea) are base materials for production of hyaluronate- collagen dressing (HCD) matrix. For the production of HCD matrix, we first dissolved the $0.1 \%$ of collagen in refined water $(\mathrm{pH} 3 \sim 4)$ and raised $\mathrm{pH}$ to $7 \sim 8$ then dissolved $0.8 \%$ of HA. The $0.1 \%$ of F68 was added to help blending collagen and HA then evenly mixed them with homogenizer. Subsequently, we added stabilized growth factors with concentrations of $0.1,0.3$, 1 , and $2.5 \mathrm{ug} / \mathrm{cm}^{2}$ and aliquot them into the mold for the lyophilization (Additional file 1: Figure S1). For selection of optimal sterilization method of S-EGF and S-bFGF, we exerted various kinds of methods following ethylene oxide (EO) gas, gamma irradiation (25 kGy) and electronic irradiation. The stabilized epidermal growth factor (S-EGF) and basic fibroblast growth factor (S-bFGF) became more thermostable through structural modification when compared to existing growth factors. The stabilized growth factor loaded HCD matrixes were received from GENEWEL (Seongnam, Gyeonggi-do, Korea).

\section{MTT Assay and cell proliferation assay}

L929 cells (Sigma-Aldrich, St Louis, MO, USA) were cultured in Dulbecco's Modified Eagle's Medium-high glucose (DMEM; Gibco, Carlsbad, CA, USA) supplemented with $10 \% \mathrm{FBS}$ and $1 \%$ penicillin/streptomycin (P/S). The NIH/3T3 fibroblast cells (Sigma-Aldrich) and Balb/3T3 fibroblast cells (Sigma-Aldrich) were cultured in DMEM (Gibco) supplemented with $0.5 \%$ bovine calf serum (BCS) and $1 \% \mathrm{P} / \mathrm{S}$. These cells were incubated at $37{ }^{\circ} \mathrm{C}$ incubator with $5 \% \mathrm{CO}_{2}$ and medium was replenished every two days. Each of cells were seeded at density of $1 \times 10^{5}$ cells/well on 96 well plates and incubated for $24 \mathrm{~h}$. We classified the groups as negative control (no treatment), positive control (latex glove extracted solution; ISO-10993-5) [23], S-EGF, S-bFGF, and HCD matrix containing S-EGF and S-bFGF and added them in medium and cultured for $48 \mathrm{~h}$. The S-EGF and S-bFGF are loaded on HCD matrix with the concentration of $0.1,0.3,1$, and $2.5 \mu \mathrm{g} / \mathrm{cm}^{2}$. Then thyiazolyl blue tetrazolium bormide (MTT, M2128-100MG, Sigma-Aldrich) was added and incubated for $3 \mathrm{~h}$. At the indicated time, DMSO was added and absorbance was read at $570 \mathrm{~nm}$.

\section{Agar overlay}

L929 cells were seeded $4 \times 10^{5}$ cells/well on 6 well plate. After $24 \mathrm{~h}$ of cultivation, medium were removed and $1.5 \%$ agar (BD, Franklin Lakes, NJ, USA) diluted in distilled water was overlaid on the cells. After solidification of agar, $0.01 \%$ neutral red vital dye (Sigma-Aldrich) was treated on medium and placed in dark $37{ }^{\circ} \mathrm{C}$ incubator for $90 \mathrm{~min}$. The sterilized paper discs $(6 \mathrm{~mm})$ containing $\mathrm{HA}$, were laid on agar and incubated in dark for $24 \mathrm{~h}$. After incubation, cytotoxicity was rated with decolorized zone around the paper discs [24].

\section{Enzyme-Linked Immunosorbent Assay (ELISA)}

Each of disk-shaped HCD matrix containing $1 \mu \mathrm{g} / \mathrm{cm}^{2}$ of S-EGF and S-bFGF (diameter of $10 \mathrm{~mm}$ ) was immersed in $10 \mathrm{ml}$ of $0.1 \%$ BSA (Sigma-Aldrich) dissolved in PBS and incubated in shaking incubator at $37{ }^{\circ} \mathrm{C}$ with $15 \mathrm{rpm}$ for 7 days. Thereafter, to determine the amount of growth factors, these mixtures were diluted to $1 / 1000$ and analyzed by ELISA kit (R\&D system, St Cloud, MN, USA). Also, to confirm concentration of released S-EGF and S-bFGF from HCD matrix, we installed matrices (diameter of $30 \mathrm{~mm}$ ) in the Franz Cell and 
incubated in shaking incubator at $37{ }^{\circ} \mathrm{C}$ with $50 \mathrm{rpm}$. Then, collected samples on day 1, 3, 7, 10, 14, 21 and confirmed quantity of S-EGF and S-bFGF with ELISA kit (R\&D system).

\section{Preparation of streptozotocin (STZ)-induced type1 diabetic mouse model}

ICR mice (Male, aged 7 weeks) were purchased from Orient Bio (Seongnam, Gyeonggi-do, Korea) and housed in wire cage at $20-22{ }^{\circ} \mathrm{C}$ at a relative humidity of $40-50 \%$. We used type1 diabetic mice, induced by intraperitoneal (I.P.) injection of streptozotocin (STZ) $(200 \mathrm{mg} / \mathrm{kg}$ body weight; Sigma-Aldrich) dissolved in $0.05 \mathrm{M}$ citrate buffer (pH4.5). One week after induction of diabetes, blood glucose levels were measured using OneTouch Select meter (Johnson\&Johnson, New Brunswick, NJ, USA). The diabetic phenotype in animals was confirmed by blood glucose levels over $300 \mathrm{mg} / \mathrm{dL}$ and maintained for three weeks. The animal experiments were carried out in accordance with guidelines set by the Department of Laboratory Animal Resources, Yonsei University College of Medicine and Seoul, Korea (Permit number = 2015-0190).
Biocompatibility test of S-EGF and S-bFGF loaded on HCD matrix in type1 diabetic mouse model

STZ-induced type1 diabetic mice (Male, aged 9 weeks) were anesthetized with I.P. injection of Zoletile $(30 \mathrm{mg} /$ $\mathrm{kg}$ body weight) and Rumpon (10 mg/kg body weight). The hair on the back of mouse was shaved and subsequently wiped with $70 \%$ ethanol. A $10 \mathrm{~mm}$ diameter full-thickness of skin wounds were created on the backs of STZ-induced diabetic mice and fixed with a silicone ring to prevent wound contraction. The groups are classified as Defect control, HCD only, HCD+S-EGF $0.3 \mu \mathrm{g} / \mathrm{cm}^{2}, \mathrm{HCD}+\mathrm{S}-\mathrm{EGF} 1 \mu \mathrm{g} / \mathrm{cm}^{2}, \mathrm{HCD}+\mathrm{S}-\mathrm{EGF}$ $2.5 \mu \mathrm{g} / \mathrm{cm}^{2}, \mathrm{HCD}+\mathrm{S}-\mathrm{bFGF} 0.3 \mu \mathrm{g} / \mathrm{cm}^{2}, \mathrm{HCD}+\mathrm{S}-\mathrm{bFGF}$ $1 \mu \mathrm{g} / \mathrm{cm}^{2}, \mathrm{HCD}+\mathrm{S}$-bFGF $2.5 \mu \mathrm{g} / \mathrm{cm}^{2}$. After application of matrix on wound site, Vaseline gauze (Covidien, St. Louis, MO, USA) and Neo dressing (Everaid, Gangnam, Seoul, Korea) were placed on matrix for the minimal dehydration of wound sites. In each groups, twelve animals were used.

\section{Statistical analysis}

Statistical analysis was performed via Student's T-TEST and ANOVA. The data was expressed as the mean \pm
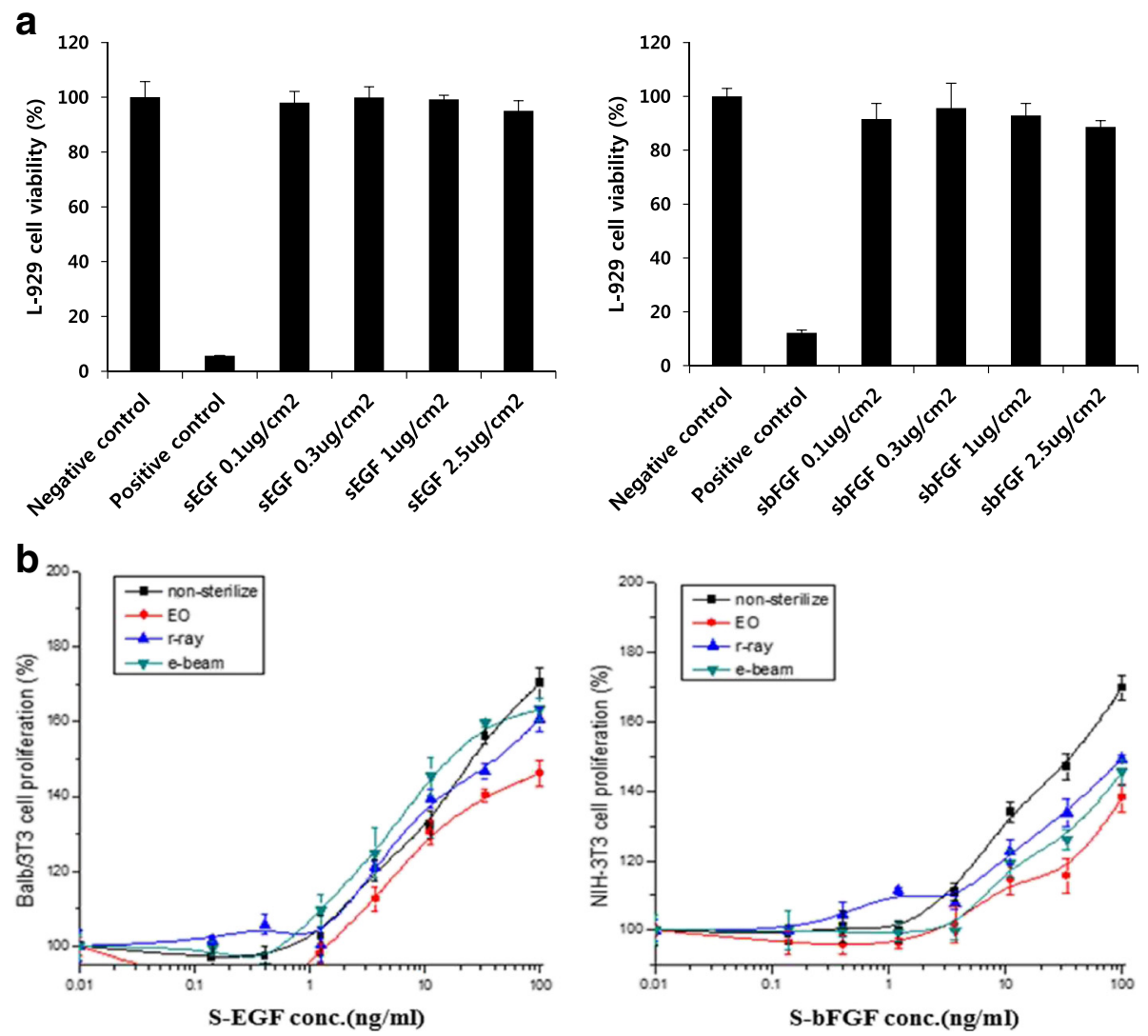

Fig. 1 The cytotoxicity stabilized growth factors were tested with various concentrations; $0.1 \mu \mathrm{g} / \mathrm{cm}^{2}, 0.3 \mu \mathrm{g} / \mathrm{cm}^{2}, 1 \mu \mathrm{g} / \mathrm{cm}^{2} \mathrm{and} 2.5 \mu \mathrm{g} / \mathrm{cm}^{2}(\mathbf{a})$ and proliferation rate of S-EGF and S-bFGF after sterilization via EO gas, gamma irradiation and electron irradiation (b) 

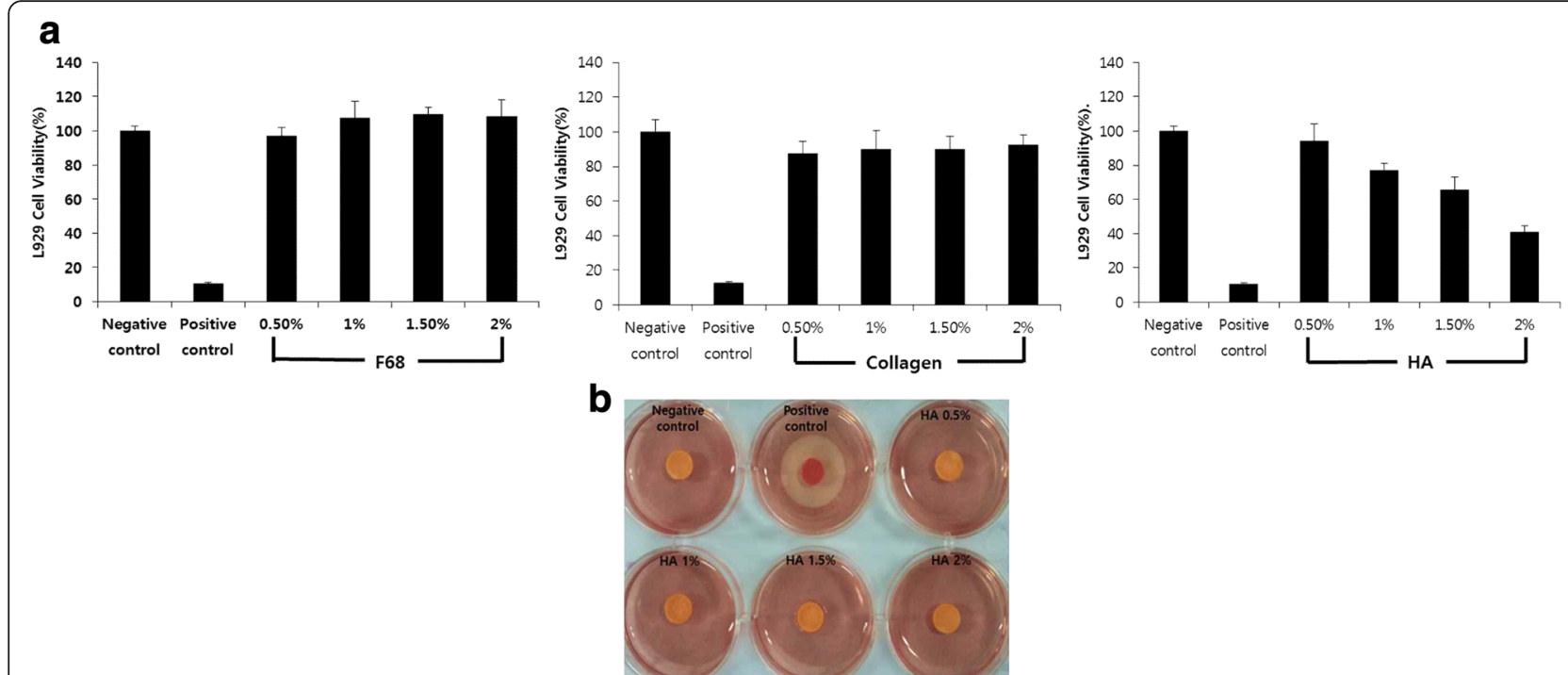

Fig. 2 The biocompatibility of base materials of HCD matrix, in vitro, was confirmed by cytotoxicity assay of base materials such as F68, collagen and HA (a) and the HA was confirmed by agar overlay with various percentages; $0.5 \%, 1 \%, 1.5 \%$, and $2 \%$ (b)

standard deviation (SD). Values of $* p<0.05, * * p<0.01$ were considered statistically significant.

\section{Results and discussion}

\section{Biological stability of S-EGF and S-bFGF}

First, we performed cytotoxicity assay for the evaluation of the biological stability of stabilized epidermal growth factor (S-EGF) and basic fibroblast growth factor (SbFGF). All concentrations of S-EGF and S-bFGF have no significance compared to negative control, indicating that S-EGF and S-bFGF do not retain toxicity (Fig. 1a). The result of sterilization, the gamma and electron irradiated S-EGF and S-bFGF showed relatively high proliferation rate compared to EO gas (Fig. 1b). We selected gamma irradiation as our sterilization method because it is better to assure product sterility and also more penetrating than electronic irradiation [25]. The S-EGF and S-bFGF were biologically stable.

\section{Biological safety of HCD matrix}

For biocompatibility of HCD matrix, we evaluated cytotoxicity of three base materials, respectively. The collagen and F68 had more than $90 \%$ of cell survival rate, whereas more than $1 \%$ of HA showed reduction of survival rate (Fig. 2a). Since the cytotoxicity of HA appears to be the characteristics of viscoelasticity rather than cytotoxicity $[26,27]$, we substituted MTT assay to agar
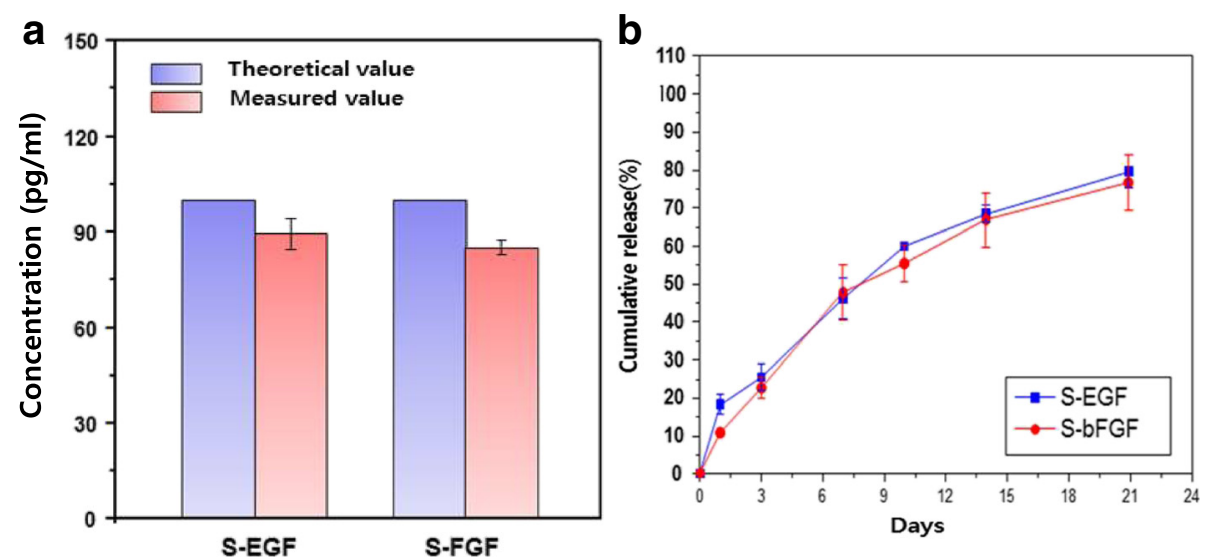

Fig. 3 Characteristics of S-EGF and S-bFGF loaded HCD matrix. Amount of S-EGF and S-bFGF contained in HCD matrix was measured via ELISA (a) and cumulative release of S-EGF and S-bFGF that are loaded on HCD matrix was measured by ELISA during 21 days (b) 
overlay test that is minimally influenced by viscoelasticity. All concentrations of HA showed no decolorized zones, suggesting that HA had no toxicity (Fig. 2b). These data indicate that there is no cytotoxicity in $\mathrm{HCD}$ matrix as base material.

The capacity of S-EGF and S-bFGF loaded on HCD matrix, and its cytotoxicity, in vitro

We evaluated the final concentration of S-EGF and S-bFGF that are loaded on HCD matrix. As a result, the concentrations were $89.44 \pm 4.6 \mathrm{pg} / \mathrm{ml}$ and $85.27 \pm$ $2.07 \mathrm{pg} / \mathrm{ml}$, respectively (Fig. 3a). When we evaluated the releasing period of growth factors from HCD matrix, the growth factors were gradually released during long-term period until day21 (Fig. 3b). Thus, this result demonstrated the potential utility of HCD matrix as an agent delivery scaffold.

Additionally, we determined the biological stability of S-EGF and S-bFGF loaded on HCD matrix. Similar to $\mathrm{HCD}$ group as a negative control, each concentration of S-EGF and S-bFGF loaded on HCD matrix showed more than $90 \%$ of cell survival rate at all concentrations. These results define the biological safety of our S-EGF (Fig. 4a) and S-bFGF (Fig. 4b) loaded HCD matrix, suggesting the optimal biocompatibility as wounddressing material.

The stability of S-EGF and S-bFGF loaded on HCD matrix, in vivo

Next, we assessed the stability of S-EGF and S-bFGF loaded on HCD matrix in vivo. These matrixes had no inflammatory effect at day 7 , indicating that the S-EGF and S-bFGF loaded on HCD matrix is negligent as wound-dressing material. In addition, the S-EGF and SbFGF loaded on HCD matrix showed slight acceleration of wound healing when compared to defect control and HCD matrix only group (Fig. 5a). As shown in Fig. 5b, the wound areas of S-EGF group were decreased about $50 \%$ compared with defect control group (Fig. 5b). Thus, these results suggest that S-EGF and S-bFGF loaded on HCD matrix is biologically safe to use as wound-dressing material and have the potential in acceleration of wound healing.

Taken together, our results demonstrated that S-EGF and S-bFGF are more stabilized than existing EGF and bFGF after modification, resulting in longer half-life and releasing period of growth factors from HCD matrix, in vitro. Indeed, this matrix was biologically safe, in vivo. Although, the further studies toward S-EGF and S-bFGF

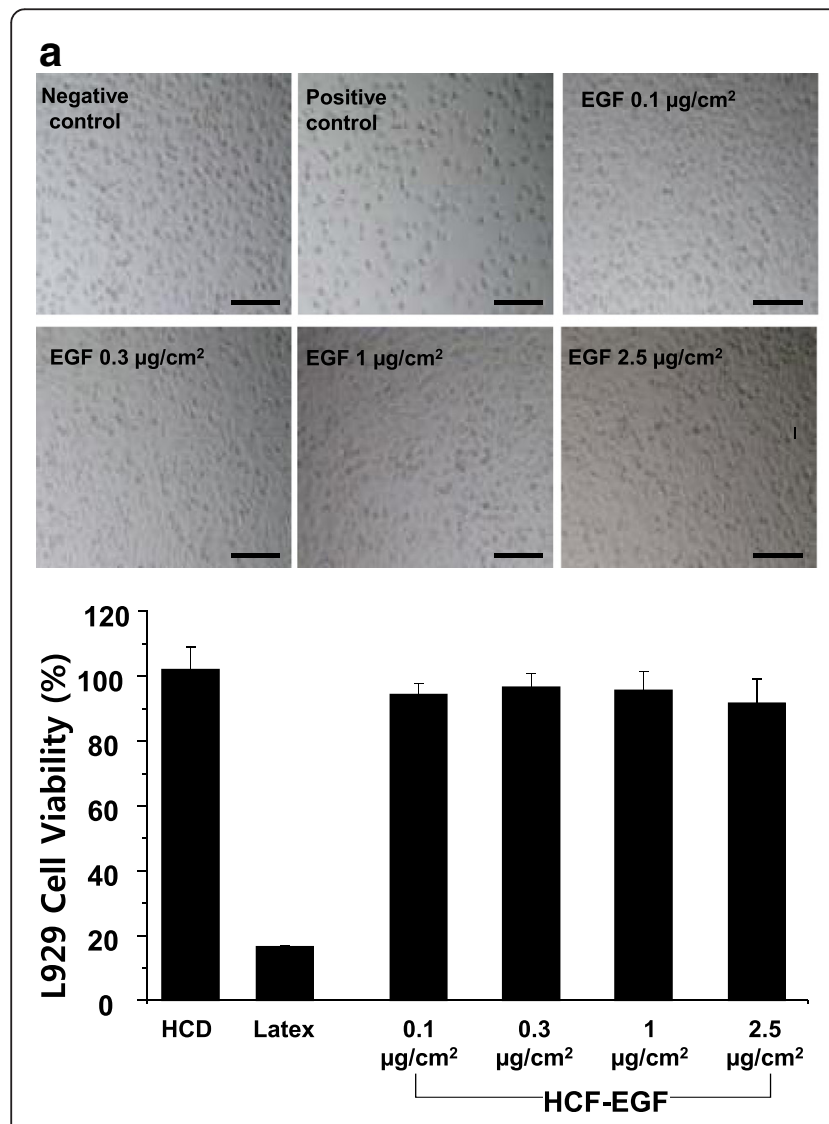

\section{b}
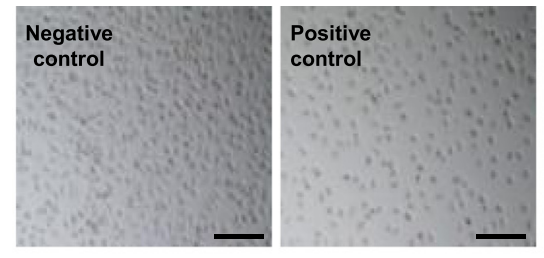

bFGF $0.1 \mu \mathrm{g} / \mathrm{cm}^{2}$
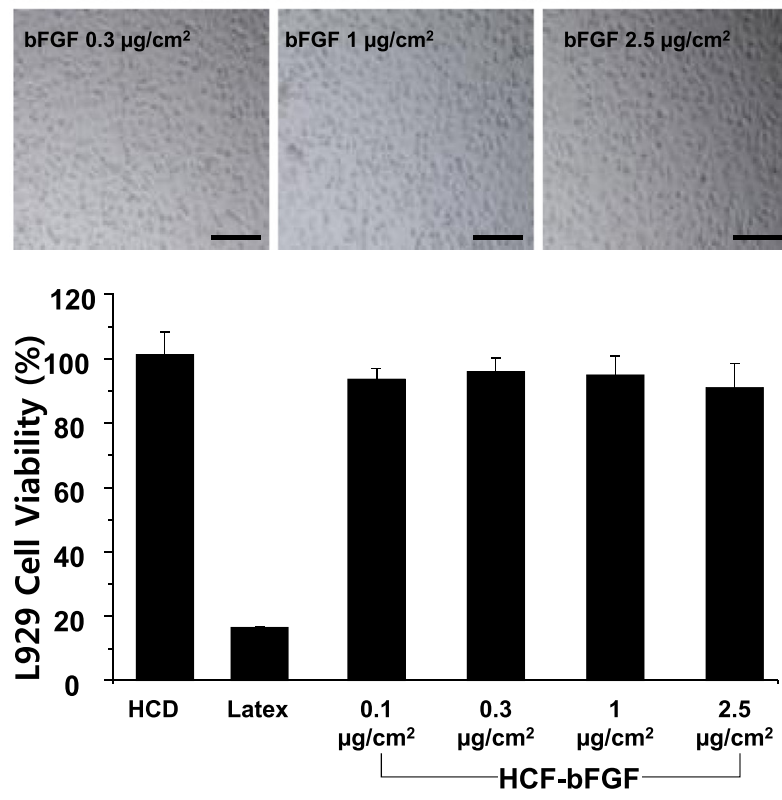

Fig. 4 Cytotoxicity of HCD matrix containing S-EGF and S-bFGF was measured with various concentrations of S-EGF (a) and S-bFGF (b). Scale bar = $200 \mu \mathrm{m}$ 


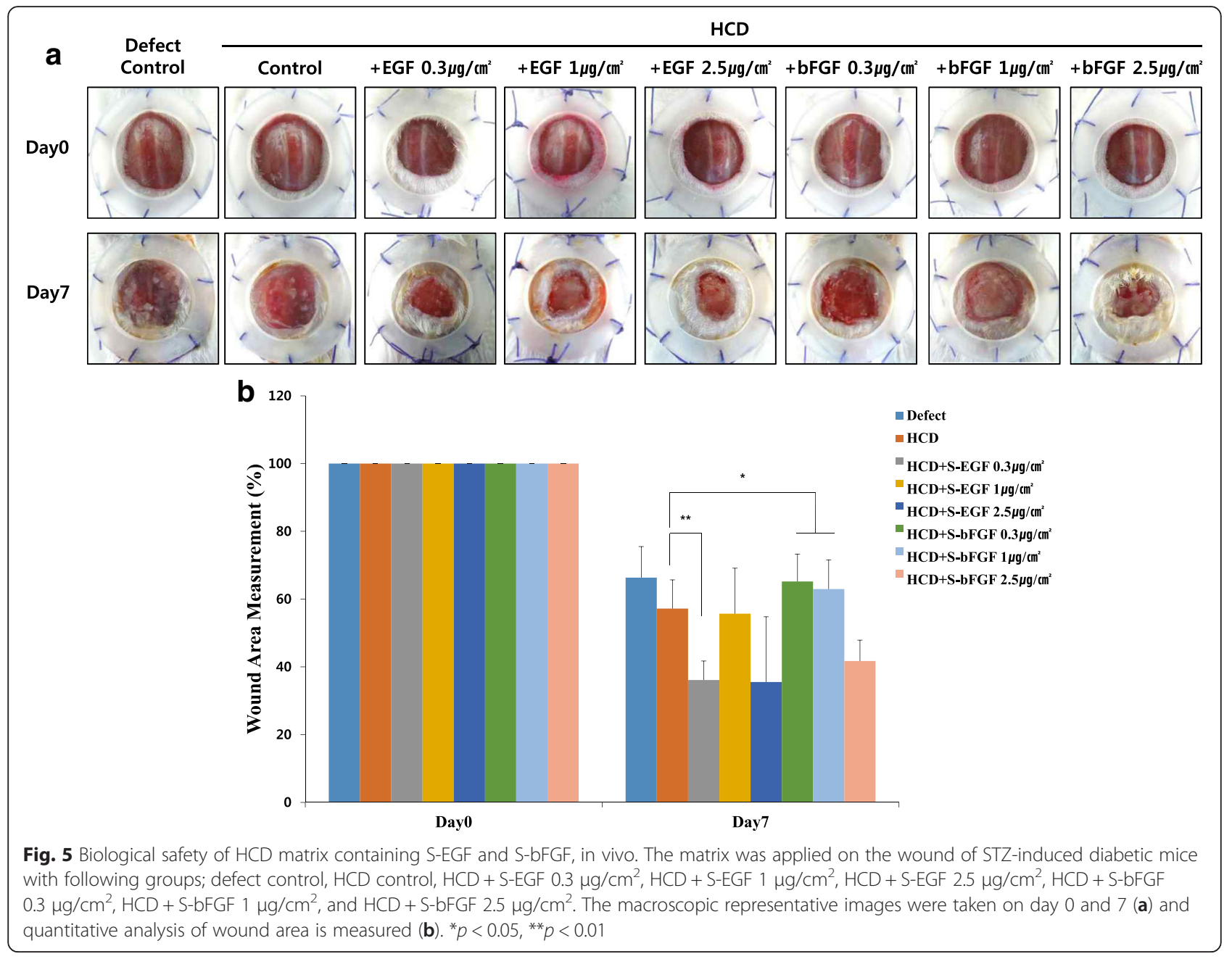

loaded on HCD matrix are needed to evaluate the effectiveness, in vivo, S-EGF and S-bFGF loaded on HCD matrix is expected to be valuable wound-dressing material in impaired wound healing.

\section{Conclusions}

For impaired wound healing, we developed the S-EGF and S-bFGF loaded on HCD matrix, which has no effects on cytotoxicity and proliferation. Also, we confirmed stability and releasing period of S-EGF and S-bFGF loaded on HCD matrix. As a result, these matrixes showed more stabilized and longer releasing period of growth factors than existing EGF and bFGF, in vitro. Furthermore, when these matrixes were applied on the wound of diabetic mice, the inflammatory response was not occurred, indicating that this matrix is biocompatible. According to these results, the S-EGF and S-bFGF loaded on HCD matrix may overcome the disadvantages of established scaffolds in wound healing study. Taken together, the SEGF and S-bFGF loaded on HCD matrix may contribute to the application in impaired wound healing because of the biological safety.

\section{Additional file}

Additional file 1: Figure S1. The fabrication process of S-EGF and S-bFGF loaded HCD matrix with collagen, HA, and F68. (PDF 42 kb)

\section{Abbreviations}

F68: pluronic 68; HA: hyaluronic acid; HCD: Hyaluronate- Collagen Dressing; S-bFGF: stabilized-basic fibroblast growth factor; S-EGF: stabilized-epidermal growth factor.

\section{Competing interests}

The authors declare that they have no competing interests.

\section{Authors' contributions}

SMC designed the experiments and wrote the manuscript. HAR and KML came up with concept and designed the experiments. HJK, IKP and WJC came up with concept and provided materials. HCS provided modified materials. WJC and JWL discussed the results and commented on the manuscript. All authors read and approved the final manuscript. 


\section{Acknowledgement}

This work was supported by Industrial Technology Innovation Program (Advanced Technology Center Program) funded by the Ministry of Trade, Industry and Energy (MOTIE, Korea) (No. 10048247: Development of chronic wound dressing load growth factor for an aging society).

\section{Author details}

'Brain Korea 21 PLUS Project for Medical Science, Yonsei University, Seoul, South Korea. ${ }^{2}$ Department of Orthopaedic Surgery, Yonsei University College of Medicine, Seoul, South Korea. ${ }^{3}$ R\&D center, Genewel co., Ltd, Sungnam, Korea. ${ }^{4}$ School of Systems Biomedical Science, Soongsil University, Seoul 156-743, Korea.

Received: 13 March 2016 Accepted: 24 March 2016

Published online: 01 April 2016

\section{References}

1. Obara K, Ishihara M, Kanatani MFY, Hattori H, Matsui T, Takase B, Ozeki Y, Nakamura S, Ishizuka T, Tominaga S, Hiroi S, Kawai T, Maehara T. Acceleration of wound healing in healing-impaired $\mathrm{db} / \mathrm{db}$ mice with a photocrosslinkable chitosan hydrogel containing fibroblast growth factor-2. Wound Rep Reg. 2005;13:390-7.

2. Greenhalgh DG, Sprugel KH, Murray MJ, Ross R. PDGF and FGF Stimulate Wound Healing in the Genetically Diabetic Mouse. Am J Pathol. 1990;136:1-6.

3. Bennet NF, Schultz GS. Growth factors and wound healing. Part II: role in normal and chronic wound healing. Am J Surg. 1993;166:74-81.

4. Cross KJ, Mustoe TA. Growth factors in wound healing. Surg Clin North Am. 2003;83:531-46.

5. Grotendorst GR, Martin GR, Pencev D, et al. Stimulation of granulation tissue formation by platelet-derived growth factor in normal and diabetic rats. J Clin Invest. 1985:76:2323-9.

6. Broadley KN, Aquino AM, Hicks B, et al. Growth factors bFGF and TGF beta accelerate the rate of wound repair in normal and in diabetic rats. Int J Tissue React. 1988;10:345-53.

7. Buckley A, Davidson JM, Kamerath TBW, et al. Sustained release of epidermal growth factor accelerates wound repair. Proc Natl Acad Sci USA. 1985;82:7340-4.

8. Werner S, Grose R. Regulation of wound healing by growth factors and cytokines. Physiological reviews. 2003;83:835-70.

9. Park SA, Teixeira LB, Raghunathan VK, Covert J, Dubielzig RR, Isseroff RR, Schurr M, Abbott NL, McAnulty J, Murphy CJ. Full-thickness splinted skin wound healing models in $\mathrm{db} / \mathrm{db}$ and heterozygous mice: implications for wound healing impairment. Wound Repair Regen. 2014;22:368-80.

10. Velnar T, Bailey T, Smrkolj V. The wound healing process: an overview of the cellular and molecular mechanisms. J Int Med Res. 2009;37:1528-42.

11. Obara K, Ishihara M, Fujita M, Kanatani Y, Hattori H, Matsui T, Takase B, Ozeki Y, Nakamura S, Ishizuka T, Tominaga S, Hiroi S, Kawai T, Maehara T. Acceleration of wound healing in healing-impaired $\mathrm{db} / \mathrm{db}$ mice with a photocrosslinkable chitosan hydrogel containing fibroblast growth factor-2. Wound Repair Regen. 2005;13:390-7.

12. Demirdogen $B$, Elcin AE, Elcin YM. Neovascularization by bFGF releasing hyaluronic acid-gelatin microspheres: in vitro and in vivo studies. Growth Factors. 2010;28:426-36.

13. Tonnesen MG, Feng $X$, Clark RA. Angiogenesis in wound healing. J Investig Dermatol Symp Proc. 2000:5:40-6.

14. Dogan S, Demirer S, Kepenekci I, Erkek B, Kiziltay A, Hasirci N, Muftuoglu S, Nazikoglu A, Renda N, Dincer UD, Elhan A, Kuterdem E. Epidermal growth factor-containing wound closure enhances wound healing in non-diabetic and diabetic rats. Int Wound J. 2009;6:107-15.

15. Simons M, Annex BH, Laham RJ, Kleiman N, Henry T, Dauerman H, Udelson JE, Gervino EV, Pike M, Whitehouse MJ, Moon T, Chronos NA. Pharmacological treatment of coronary artery disease with recombinant fibroblast growth factor-2: double-blind, randomized, controlled clinical trial. Circulation. 2002:105:788-93.

16. Rajesh K, Mark CM. The Stability Factor: Importance in Formulation Development. Curr Pharm Biotechnol. 2002;3:361-71.

17. Cohen S, Carpenter G. Human epidermal growth factor: isolation and chemical and biological properties. Proc Natl Acad Sci USA. 1975:72:1317-21.

18. Marks MG, Doillon C, Silver FH. Effects of fibroblasts and basic fibroblast growth factor on facilitation of dermal wound healing by type I collagen matrices. J Biomed Mater Res. 1991;25:683-96.
19. Obara K, Ishihara M, Ishizuka T, Fujita M, Ozeki Y, Maehara T, Saito Y, Yura H, Matsui T, Hattori H, Kikuchi M, Kurita A. Photocrosslinkable chitosan hydrogel containing fibroblast growth factor-2 stimulates wound healing in healing-impaired db/db mice. Biomaterials. 1991;24:3437-44.

20. Dogan S, Demirer S, Kepenekci I, Erkek B, Kiziltay A, Hasirci N, Muftuoglu S, Nazikoglu A, Renda N, Dincer UD, Elhan A, Kuterdem E. Epidermal growth factor containing wound closure enhances wound healing in non-diabetic and diabetic rats. Int Wound J. 2009;2:107-15.

21. Wang W, Lin S, Xiao Y, Huang Y, Tan Y, Lu C, Li X. Acceleration of diabetic wound healing with chitosan-crosslinked collagen sponge containing recombinant human acidic fibroblast growth factor in healing-impaired STZ diabetic rats. Life Sci. 2008;82(3-4):190-204.

22. Nagato H, Ymebayashi Y, Wako M, Tabata Y, Manabe M. Collagen-poly glycolic acid hybrid matrix with basic fibroblast growth factor accelerated angiogenesis and granulation tissue formation in diabetic mice. J Dermatol. 2006;33(10):670-5.

23. Kim AR, Park HS, Kim SS, Noh I. Biological Evaluation of Cellulose Hydrogel with Temperature-Responsive Particles. Biomater Res. 2013;17(4):181-6.

24. Schmalz G. Agar overlay method. Int Endod J. 1988;21:59-66.

25. Hasanain F, Guenther K, Mullett WM, Craven E. Gamma sterilization of pharmaceuticals-a review of the irradiation of excipients, active pharmaceutical ingredients, and final drug product formulations. PDA J Pharm Sci Technol. 2014;68:113-37.

26. Zhang Z, Christopher GF. The nonlinear viscoelasticity of hyaluronic acid and its role in joint lubrication. Soft Matter. 2015;11:2596-603.

27. Borzacchiello A, Russo L, Malle BM, Schwach-Abdellaoui K, Ambrosio L. Hyaluronic Acid Based Hydrogels for Regenerative Medicine Applications. BioMed Res Int. 2015:2015:1-12.

\section{Submit your next manuscript to BioMed Central and we will help you at every step:}

- We accept pre-submission inquiries

- Our selector tool helps you to find the most relevant journal

- We provide round the clock customer support

- Convenient online submission

- Thorough peer review

- Inclusion in PubMed and all major indexing services

- Maximum visibility for your research

Submit your manuscript at www.biomedcentral.com/submit
C) Biomed Central 